\title{
Sublattice dynamics and quantum state transfer of doublons in two-dimensional lattices
}

\author{
M. Bello, ${ }^{1}$ C. E. Creffield, ${ }^{2}$ and G. Platero ${ }^{1}$ \\ ${ }^{1}$ Instituto de Ciencias de Materiales de Madrid, CSIC E-28049, Spain \\ ${ }^{2}$ Departamento de Física de Materiales, Universidad Complutense de Madrid E-28040, Spain \\ (Received 2 August 2016; revised manuscript received 18 January 2017; published 7 March 2017)
}

\begin{abstract}
We analyze the dynamics of two strongly interacting fermions moving in two-dimensional lattices under the action of a periodic electric field, both with and without a magnetic flux. Due to the interaction, these particles bind together forming a doublon. We derive an effective Hamiltonian that allows us to understand the interplay between the interaction and the driving, revealing surprising effects that constrain the movement of the doublons. We show that it is possible to confine doublons to just the edges of the lattice and to a particular sublattice if different sites in the unit cell have different coordination numbers. Contrary to what happens in one-dimensional systems, here we observe the coexistence of both topological and Shockley-like edge states when the system is in a nontrivial phase.
\end{abstract}

DOI: 10.1103/PhysRevB.95.094303

\section{INTRODUCTION}

Tunneling dynamics of particles in lattices can be well understood with tight-binding Hamiltonians. In these models, quantum coherence is responsible for many exotic phenomena, such as system revivals, quantum interference, and Rabi-like oscillations. It has now become possible to observe these effects in a variety of setups ranging from photonic crystals [1-4] to quantum dots [5-7] and cold atoms trapped in optical lattices [8-11]. In particular, quantum coherence allows the transfer of quantum information between different locations, a process known in the literature as quantum state transfer (QST). Given its importance in quantum information processing applications, QST has been the object of study in many experimental and theoretical works carried out in recent years [12-15].

Adding a periodic driving potential considerably enriches the physics of these systems and provides a means for controlling and manipulating them. Such driving can produce effects, such as dynamical localization [16] and coherent destruction of tunneling (CDT) [17], and can even be used to design artificial gauge fields [18,19]. This flexibility and controllability makes driven lattice systems ideal for use as quantum simulators [20,21]. Despite the many advances in the field, however, driven interacting systems have been less studied than non-interacting ones. Understanding the role interactions play in these setups is a hard task of fundamental importance, however, since the behavior of the system may change drastically compared with the noninteracting case and produce novel and unusual physics.

Our aim in this paper is to extend QST to interacting systems of few particles. We investigate the dynamics of two strongly interacting fermions in two-dimensional (2D) lattices. The fermions can bind together repulsively, forming what is termed a "doublon," a long-lived excitation whose decay is forbidden on energetic grounds [22-24]. Bosons can also bind together in this way, and the quantum walk for repulsively bound bosonic particles on a one-dimensional (1D) lattice has recently been studied in Ref. [25]. The regime of strongly interacting particles, i.e., that of doublons rather than single particles, is interesting in itself. There are several experiments analyzing the dynamics of high-energy bound states of ultracold fermions and bosons [24,26,27].
We derive an effective Hamiltonian describing the motion of single doublons in 2D lattices coupled to circularly polarized ac fields in the presence of a magnetic flux threading the lattice. For a special class of lattices, we demonstrate an interesting effect by which the doublon's dynamics is restricted to just one of the sublattices of the crystal. Not only that, our results show it is possible to confine the doublon dynamics to certain sites (those with the same coordination number) on the edges of any finite system and induce direct transfer of the doublon between distant sites, avoiding the intervening sites. This makes the process less susceptible to decoherence. Although we present results mainly for the Lieb lattice, the conclusions we draw apply to a wide set of 2D lattices.

\section{MODEL}

We consider a Hubbard model for fermions with an external ac field and a uniform magnetic field perpendicular to the plane of the lattice. The ac field couples to the particle density, and the magnetic flux induces phases in the hoppings such that the sum of the phases around a closed loop is the total flux threading the loop, measured in units of the magnetic flux quantum $\Phi_{0}=h / e$. The system is then described by the tight-binding model,

$$
\begin{aligned}
H(t)= & -J \sum_{\langle i, j\rangle, \sigma} e^{i \phi_{i j}} c_{i \sigma}^{\dagger} c_{j \sigma}+U \sum_{i} n_{i \uparrow} n_{i \downarrow} \\
& +\sum_{i} V_{i}(t)\left(n_{i \uparrow}+n_{i \downarrow}\right),
\end{aligned}
$$

where $c_{i \sigma}^{\dagger}\left(c_{i \sigma}\right)$ is the creation (annihilation) operator of a fermion on site $i$ with spin $\sigma$ and $n_{i \sigma}=c_{i \sigma}^{\dagger} c_{i \sigma}$ is the usual number operator. We choose a circularly polarized driving: $V_{i}(t)=x_{i} E \cos (\omega t)+y_{i} E \sin (\omega t)$, where $x_{i}$ and $y_{i}$ are the coordinates of site $i$. The parameters of the model are the interaction strength $U$, the hopping amplitude $J$, the ac field amplitude $E$, and frequency $\omega$.

In the strongly interacting limit of the undriven model, particles can bind together repulsively forming a doublon [22,28,29]. This bound state consists of two particles with opposite spin occupying the same lattice site. If initially two particles form a doublon, they will remain bound together 
thereafter in the absence of dissipation. This can be understood on energetic grounds. The kinetic energy of a single particle in a lattice is limited by the width of the energy bands, which is proportional to the hopping amplitude; thus if $U \gg J$, doublons cannot decay into single particles as energy would not be conserved. In this regime, the total double occupancy is approximately a conserved quantity, and one can obtain an effective Hamiltonian for doublons by means of a SchriefferWolff transformation (SWT), projecting out single occupancy states [30].

In the presence of an ac field one might expect the stability of doublons to be spoiled. To address this question we derive an effective Hamiltonian that includes both the interaction between particles and the periodic driving using the so-called high-frequency expansion (HFE). This method allows the effective Hamiltonian to be written as a power series in $1 / \omega$, the different terms being functions of the Fourier components of the original time-periodic Hamiltonian (1) [31]. A different effective Hamiltonian is obtained depending on whether the system is in the strongly interacting regime $(U \gg \omega>J)$ or the high-frequency regime $(\omega \gg U>J)$. In the first case it corresponds to first performing the SWT and then a hopping renormalization, whereas in the second it is the other way around $[32,33]$. In the strongly interacting regime, the driving can induce the formation and dissociation of doublons. These processes involve the absorption and emission of photons with a probability amplitude proportional to $\mathcal{J}_{l}(2 E \delta / \omega)$ [33]. Thus, for small driving amplitudes, $2 E \delta<\omega l=U$ (where $\delta$ is the distance between neighboring sites and $l$ is the order of resonance), the probability is very small, and doublons persist in time. Conversely, for high driving amplitudes the total double occupancy of any given state changes considerably within a period. Up to first order, the effective Hamiltonian we find for the strongly interacting regime with small driving amplitudes is

$$
H_{\mathrm{eff}}=J_{\mathrm{eff}} \sum_{\langle i, j\rangle} e^{i 2 \phi_{i j}} d_{i}^{\dagger} d_{j}+\sum_{i} \mu_{i} n_{i}^{d},
$$

where $d_{i}^{\dagger}=c_{i \uparrow}^{\dagger} c_{i \downarrow}^{\dagger}\left(d_{i}\right)$ is the creation (annihilation) operator of a doublon on site $i$ and $n_{i}^{d}=d_{i}^{\dagger} d_{i}$ is the doublon number operator. In this result, additional terms including the interaction between doublons have been neglected as we consider the dynamics of just a single doublon. Also, following the previous reasoning, we have neglected terms which correspond to transitions between single-occupancy and double-occupancy states caused by the driving. $J_{\text {eff }}$ and $\mu_{i}$ can be written in terms of the original parameters as

$$
J_{\text {eff }}=\frac{2 J^{2}}{U} \mathcal{J}_{0}\left(\frac{2 E \delta}{\omega}\right), \quad \mu_{i}=\frac{2 J^{2}}{U} z_{i},
$$

where $z_{i}$ is the coordination number (the number of nearest neighbors) of site $i$. This dependence of the local effective chemical potential on the number of neighbors comes from the second-order process where the doublon splits, one of the particles remaining in the original site and the other one moving to one of its neighbors, and then recombines again in the original site. The process may involve any of the neighbors, so the total effect is an effective chemical potential proportional to the coordination number. The effective hopping amplitude
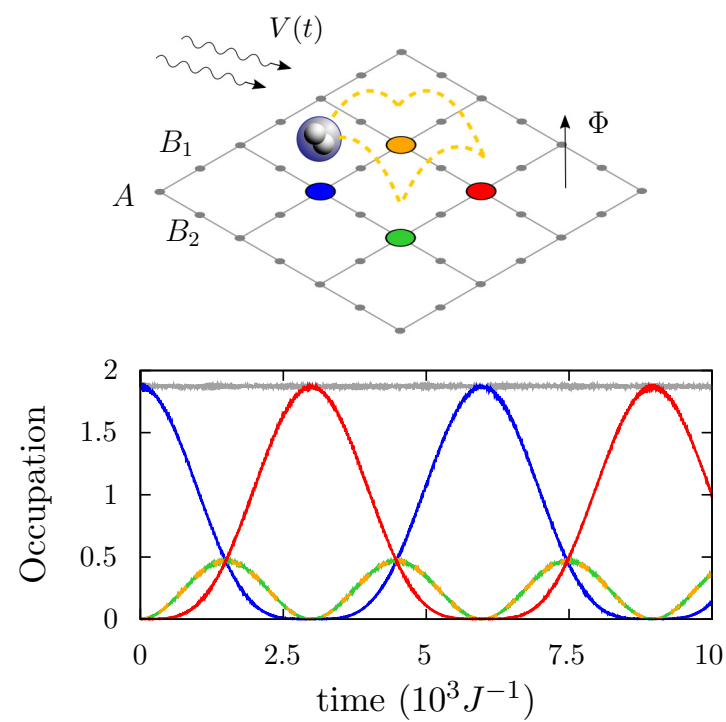

FIG. 1. (Top) Scheme depicting a doublon propagating in a finite piece of the Lieb lattice under the effect of an ac field and an external magnetic field perpendicular to the lattice. Using a periodic driving it is possible to control a doublon's effective hopping rate independent of the effective local chemical potential. Close to the CDT condition, its dynamics becomes restricted to the particular sublattice where it initially was located (colored sites), the occupation of the other sites being almost zero during the entire time evolution. (Bottom) Example of sublattice dynamics. Time evolution is obtained by numerical integration using Hamiltonian (1) parameters: $\Phi=0, U=$ $16 J, \omega=2 J$, and $E=4.8 \mathrm{~J} / \mathrm{a}$. The sum of the occupancies on the colored sites (gray line) barely varies, staying close to 2 .

for the doublon is proportional to the zeroth-order Bessel function of the first kind, whose argument depends on the parameters of the ac field and the geometry of the lattice. This hopping renormalization is isotropic because the ac field polarization is circular. A generalization for other polarizations is straightforward, but they lead to more complicated effective models.

\section{SUBLATTICE DYNAMICS}

As we can see in (3), the ac driving allows us to independently tune the effective hopping parameter with respect to the effective local potential. This has a big impact on the dynamics of doublons in lattices that can be divided into sublattices with different coordination numbers, such as the Lieb lattice shown in Fig. 1 and the $\mathcal{T}_{3}$ lattice [34,35] shown in Fig. 4. In both these examples, the effective Hamiltonian in momentum space in the absence of an external magnetic flux can be expressed as $H_{\text {eff }}=\sum_{\mathbf{k}} \Psi_{\mathbf{k}}^{\dagger} \mathcal{H}(\mathbf{k}) \Psi_{\mathbf{k}}$ with

$$
\mathcal{H}(\mathbf{k})=\left(\begin{array}{ccc}
\Delta \mu & f_{1}(\mathbf{k}) & f_{2}(\mathbf{k}) \\
f_{1}^{*}(\mathbf{k}) & 0 & 0 \\
f_{2}^{*}(\mathbf{k}) & 0 & 0
\end{array}\right) .
$$

$\Psi_{\mathbf{k}}=\left(d_{A, \mathbf{k}}, d_{B_{1}, \mathbf{k}}, d_{B_{2}, \mathbf{k}}\right)^{T}, d_{A, \mathbf{k}}$ is the annihilation operator of a doublon with quasimomentum $\mathbf{k}$ in sublattice $A$; we define $d_{B_{1}, \mathbf{k}}$ and $d_{B_{2}, \mathbf{k}}$ analogously. Its eigenvalues and eigenvectors 
(a)

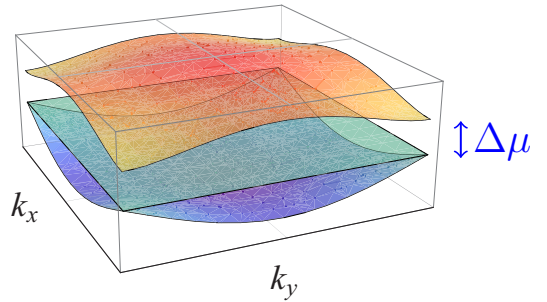

(b)

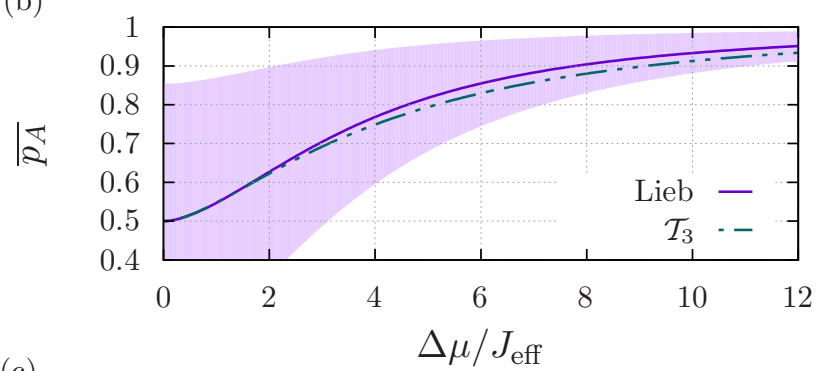

(c)

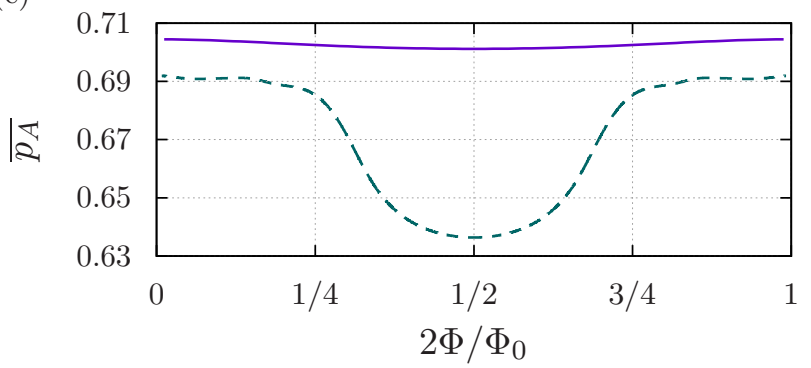

FIG. 2. (a) Energy bands for the Lieb lattice. The effective local potential experienced by the doublon opens a gap in its energy spectrum. The ac driving allows the band width to be reduced flattening the bands while keeping the gap the same. This changes the relative weight in sublattices $A$ and $B$ of the Bloch states corresponding to the upper and lower bands, see Eq. (8). (b) Calculation of the time-averaged probability to remain in sublattice $A$ for the Lieb and the $\mathcal{T}_{3}$ lattices with zero magnetic

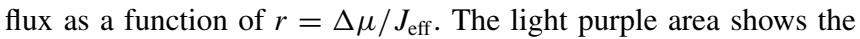
value of $\sigma_{A}$ above and below the mean for the Lieb lattice. Clearly as $\Delta \mu / J_{\text {eff }}$ increases, the effectiveness of the sublattice confinement also grows. (c) Graph of $\overline{p_{A}}$ as a function of the magnetic flux threading the elementary plaquette for $r=3$. A much stronger dependence is observed for the $\mathcal{T}_{3}$ than for the Lieb lattice. When the magnetic flux is not zero, the calculation is more involved since it is necessary to take into account the larger magnetic unit cell.

are as follows:

$$
\begin{gathered}
\epsilon_{0}(\mathbf{k})=0 \\
\epsilon_{ \pm}(\mathbf{k})=\left(\Delta \mu \pm \sqrt{4\left|f_{1}(\mathbf{k})\right|^{2}+4\left|f_{2}(\mathbf{k})\right|^{2}+\Delta \mu^{2}}\right) / 2 \\
\left|u_{\mathbf{k}}^{0}\right\rangle=\frac{1}{N}\left(0,-\frac{f_{1}(\mathbf{k})}{f_{2}(\mathbf{k})}, 1\right) \\
\left|u_{\mathbf{k}}^{ \pm}\right\rangle=\frac{1}{N}\left(\frac{\epsilon_{ \pm}(\mathbf{k})}{f_{2}^{*}(\mathbf{k})}, \frac{f_{1}^{*}(\mathbf{k})}{f_{2}^{*}(\mathbf{k})}, 1\right) .
\end{gathered}
$$

Here $N$ is just a normalization constant. Note how the states of the flat band do not have weight on the $A$ sites of the lattice [2]. We present the energy bands for the Lieb lattice in
TABLE I. Functions characterizing the energy bands of the Lieb and $\mathcal{T}_{3}$ lattices.

\begin{tabular}{lcc}
\hline \hline & $f_{1}(\mathbf{k})$ & $f_{2}(\mathbf{k})$ \\
\hline $\mathcal{T}_{3}$ & $J_{\text {eff }}\left[e^{-i\left(k_{x}+k_{y} / \sqrt{3}\right) a / 2}+e^{i\left(k_{x}-k_{y} \sqrt{3}\right) a / 2}+e^{i k_{y} a / \sqrt{3}}\right]$ & $f_{2}(\mathbf{k})=f_{1}^{*}(\mathbf{k})$ \\
Lieb & $2 J_{\text {eff }} \cos \left(k_{x} a / 2\right)$ & $2 J_{\text {eff }} \cos \left(k_{y} a / 2\right)$ \\
\hline \hline
\end{tabular}

Fig. 2(a), which clearly shows the band splitting produced by the chemical potential difference between the two sublattices $\Delta \mu=2 J^{2}\left(z_{A}-z_{B}\right) / U$. The functions $f_{1}$ and $f_{2}$ depend on the particular lattice geometry as shown in Table I. They are proportional to $J_{\text {eff }}$, which can be tuned by the ac driving. In particular, the relative weight on the $A$ sublattice of the Bloch states corresponding to the upper (lower) band can be increased (reduced) by tuning the ac field parameters closer to the CDT condition.

When studying quantum walks [36], i.e., the coherent evolution of particles in networks, it is natural to ask about the probability of finding a particle that was initially on site $i$ to be on site $j$ after a certain time $t$, that is, $p_{i j}(t)=|\langle i|U(t)| j\rangle|^{2}=$ $\left|\left\langle i\left|e^{-i H t}\right| j\right\rangle\right|^{2}$. Using (2) as the effective single-particle Hamiltonian for the doublon, we define $p_{A}(t)=\frac{1}{N_{A}} \sum_{i, j \in A} p_{i j}(t)$, which is the probability for the doublon to remain in sublattice $A$ at time $t$. To demonstrate sublattice confinement, we can compute the long-time average $\overline{p_{A}}$ and variance $\sigma_{A}^{2}=\overline{\left(p_{A}^{2}\right.}-$ ${\overline{p_{A}}}^{2}$ ), Fig. 2(b), see Appendix B. Their values are mainly determined by the ratio: $r=\Delta \mu / J_{\text {eff }}$. As shown in Fig. 2(b), the probability $\overline{p_{A}}$ can be enhanced by tuning $r$ to larger values, meaning that it is possible to confine the doublon's dynamics to a single sublattice by suitably changing the ac field parameters [see Eq. (3)]. The variance of this average probability also reduces when going in this direction. We have also computed the dependence of $\overline{p_{A}}$ with the magnetic flux threading the unit cell, see Fig. 2(c); however, its variation turns out to be minor with $\overline{p_{A}}$ gently increasing as the flux is tuned away from $2 \Phi / \Phi_{0}=1 / 2$. A much stronger dependence is observed for the $\mathcal{T}_{3}$ than for the Lieb lattice. This is to be expected as Aharonov-Bohm phases have more dramatic effects in the $\mathcal{T}_{3}$ lattice, notably the caging effect that occurs for a magnetic flux $\Phi / \Phi_{0}=1 / 2$ in the single-particle case [28,35,37].

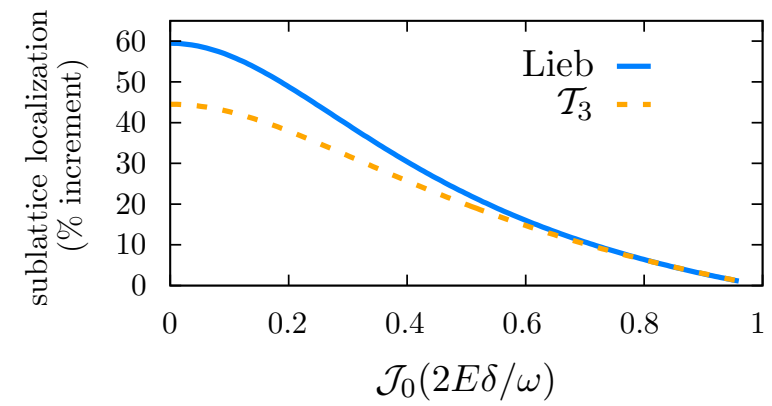

FIG. 3. Sublattice localization enhancement as a function of the hopping renormalization due to the ac driving. The abscissa corresponds to the value of $\left(\overline{p_{A}}-\overline{p_{A}}\right) / \overline{p_{A}}$, where $\overline{p_{A}} 0$ is the time-averaged probability to remain in sublattice $A$ when there is no ac driving. 

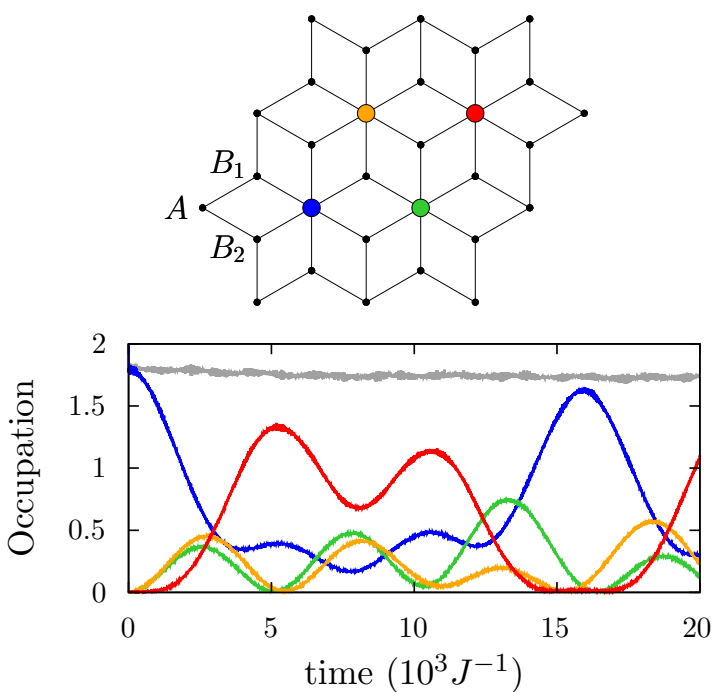

FIG. 4. Time evolution for a finite piece of the $\mathcal{T}_{3}$ lattice (see the upper scheme). The parameters of the system are as follows: $U=16 J, \Phi=0, \omega=2 J$, and $E=4.2 J / a$. The initial condition is two electrons with opposite spin occupying the lower-left site (blue curve). The average occupation on the sites not shown never exceeds the value of 0.02 per site. The doublon mostly remains in the sublattice where it initially was located. The gray line shows the sum of the occupancies of the four colored sites of the scheme.

From this analysis, it is clear that by tuning the ac field parameters closer to the CDT condition (i.e., when $2 E \delta / \omega$ is a zero of $\mathcal{J}_{0}$ ) one can enhance the confinement to the sublattice at the expense of slowing down the dynamics, see Fig. 3. Examples of sublattice dynamics are shown in Figs. 1 and 4.

\section{EDGE DYNAMICS AND QST}

In light of the effective Hamiltonian we have derived, new effects particular to systems with boundaries can be predicted. The sites on the edges of a finite lattice necessarily have fewer neighbors than those in the bulk and therefore have a smaller chemical potential [Eq. (3)]. This produces eigenstates localized on the edges, which are of the usual Shockley or Tamm type. As a consequence the doublon's dynamics can be confined to just the edges. We show an example of edge confinement in Fig. 5. Importantly, this effect is general in the sense that it happens in any kind of lattice, see Fig. 6. The resulting dynamics strongly depends on the particular shape of the boundary and the initial condition as different sites of the edge can have different numbers of neighbors. In some cases the direct transfer of doublons between distant sites of the boundary can happen as shown in Fig. 5. This occurs via the hybridization of the edge states on opposite edges, forming bilocalized eigenstates that give rise to Rabi-like oscillations. The transfer time increases exponentially with the number of sites that separate one edge from the other.

\section{TOPOLOGICAL EDGE STATES FOR DOUBLONS}

When comparing our effective model (2) to that corresponding to a Chern insulator, the only difference is the
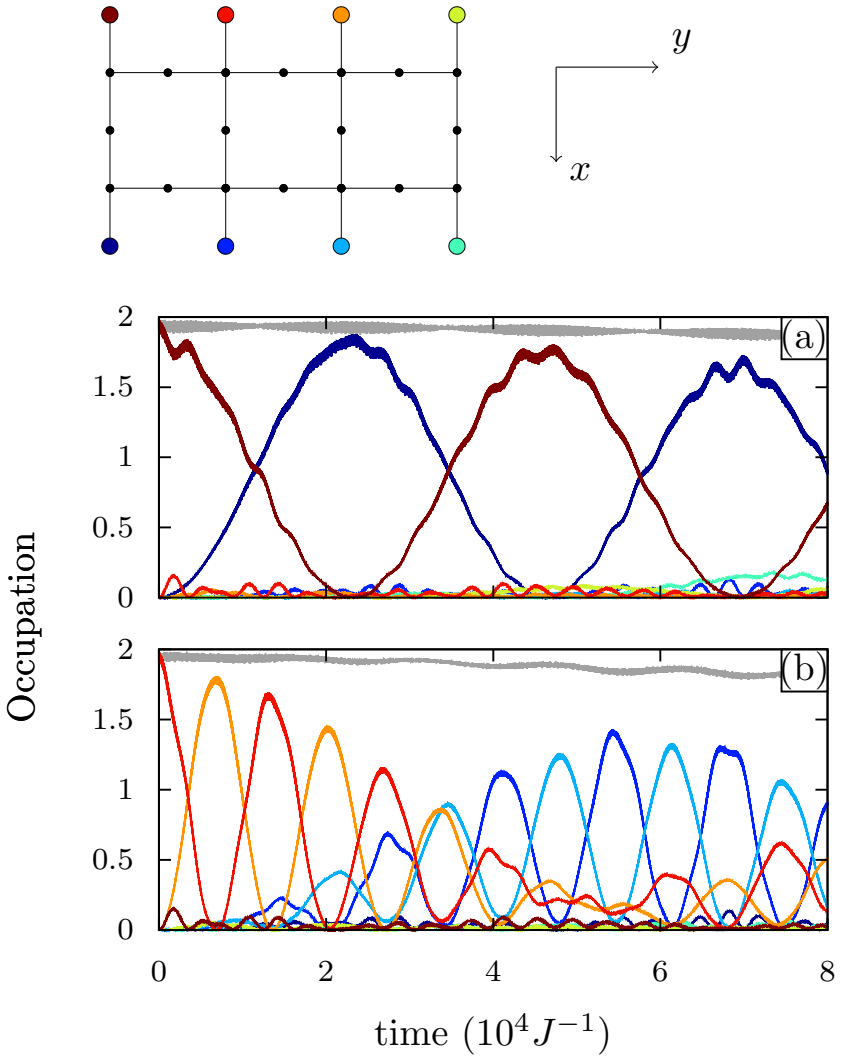

FIG. 5. Time evolution for a finite piece of the Lieb lattice, shown in the upper panel. $U=16 J, \omega=2 J$, and $E=4 J / a$. (a) Initially the doublon occupies the top-left site (in brown). The doublon oscillates between the top-left and bottom-left sites of the lattice. This oscillation is transferred to the right-top and bottom sites over a longer time period. (b) The initial condition is now a doublon occupying the middle-top site (in red). The doublon propagates mainly through the top and bottom edges. It performs oscillations between the middle sites, and on a larger time scale it is transferred to the opposite edge without occupying the intermediate sites in the bulk; this is a long-range transfer process. The occupation on the intervening sites never exceeds a value of 0.012 per site. The gray line gives the sum of the occupancies in all eight sites of the top and bottom edges.

local chemical potential term $[38,39]$. It is well known that strong disorder potentials eventually destroy the topological properties of Chern insulators as they transition to a trivial Anderson insulator by a mechanism known as "levitation and annihilation" of extended states [40,41]. Nonetheless, the chemical potential term (3) constitutes a very particular form of disorder that does not affect the topology of the system. This is in contrast to the much more drastic effect it has in 1D topological models, such as the Su-Schrieffer-Heeger model where it breaks the particle-hole symmetry needed to obtain a phase other than the trivial one [32].

To analyze the effect of the magnetic flux on the doublon's dynamics, we choose a vector potential $\mathbf{A}=B y \mathbf{u}_{x}$ corresponding to the Landau gauge and study a system periodic in the $x$ direction but finite in $y$. Interestingly, we observe in Figs. 7(a) and 7(b) that the energy spectrum shows the 

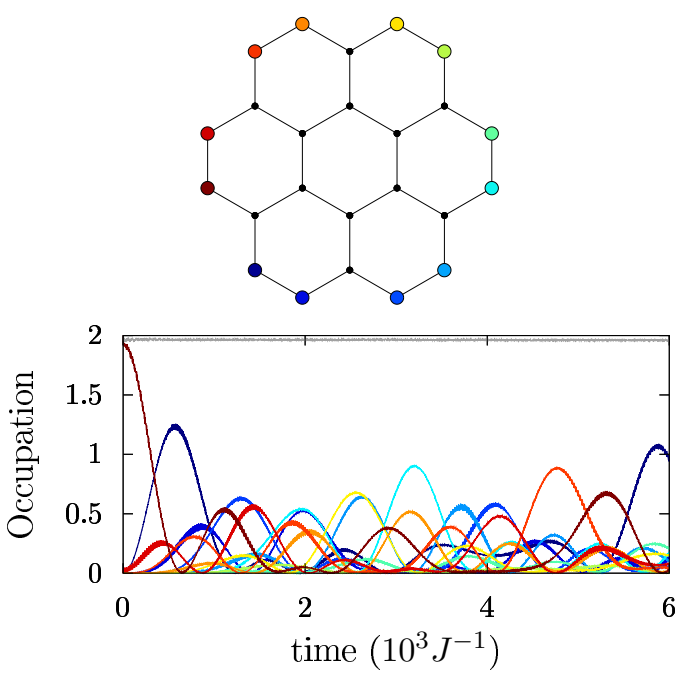

FIG. 6. Time evolution for a finite piece of the honeycomb lattice (see the upper scheme). The parameters of the system are as follows: $U=16 J, \Phi=0, \omega=2 J$, and $E=4.2 J / a$. The doublon is initially occupying the edge site in brown. It propagates mainly through the sites at the edge with only two neighbors. The average occupation on the sites not shown never exceeds the value of 0.005 per site. In gray, the total occupancy on the edge (marked sites).

coexistence of both chiral topological edge states and nonchiral Shockley-like edge states.

Furthermore, in narrow ribbons the topological edge states can also hybridize, enabling the transfer of the doublon between the two edges of the ribbon as we saw previously for the Shockley edge states. Looking at the energy spectrum, we can observe values of $k_{x}=k_{0}$ for which there are anticrossings between the edge states. At those values of momentum, a probability density initially peaked around one of the edges of the ribbon will oscillate between the two edges, while being almost equal to zero in the bulk, see Fig. 7(c).

In this paper we have concentrated mainly on the effect of the circular shaking term $V(t)$ with the magnetic flux taken as a given. A variety of techniques now exists to produce the Peierls phases in cold-atom experiments, such as the photon-assisted tunneling schemes described in Refs. [21,27], implementations based on assisted Raman transitions [42,43], or by using Berry phases to mimic the Peierls phases. One exciting possibility would be to produce the Peierls phases also by shaking so that the entire effective Hamiltonian would be produced by periodic driving. Early works on generating gauge fields on a lattice via periodic shaking were restricted to producing staggered fluxes on triangular lattices [20]. Obtaining a uniform field (of the type that we require in our system) on a lattice in which the plaquettes have parallel sides, such as the square lattice and Lieb lattice, is a much more involved problem, which requires special treatment, such as "split driving" [19].

\section{CONCLUSIONS}

We have analyzed the dynamics of two strongly interacting fermions in 2D lattices. A special property of the doublon is that it experiences a local chemical potential that depends on the coordination number of the lattice site. We propose the use
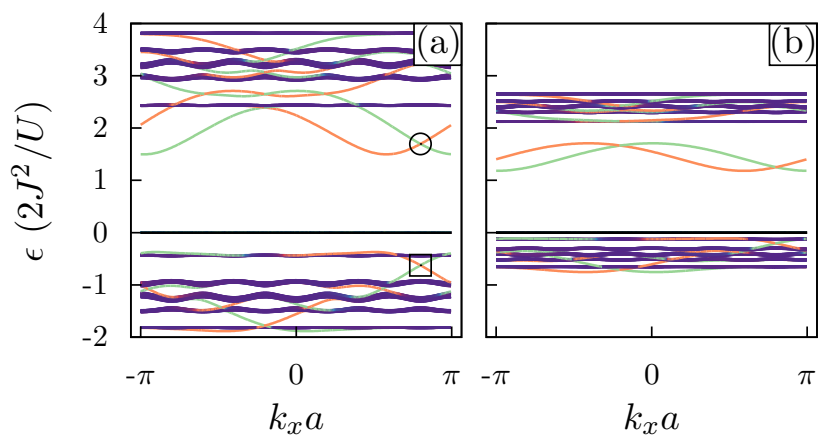

(c)
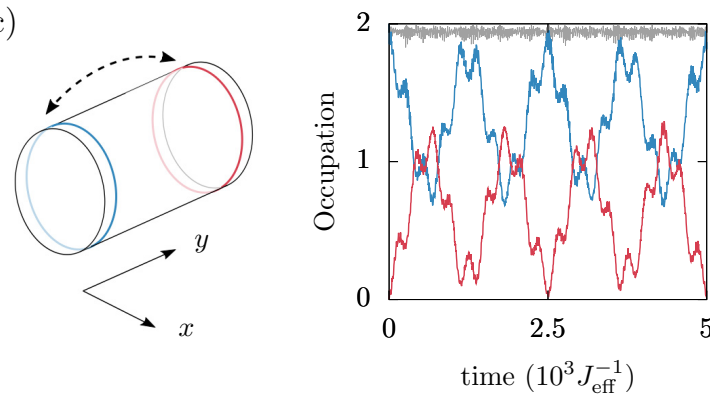

FIG. 7. (a) Energy spectrum of the undriven effective Hamiltonian for a doublon in a Lieb lattice ribbon with $N_{y}=100$ unit cells in the $y$ direction. $\Phi / \Phi_{0}=1 / 10$. The color indicates the average position in the $y$ direction of the eigenstate. Orange and green correspond to localized states on the left and right edges, respectively, whereas purple corresponds to extended bulk states. At $k_{x}=k_{0}=4 \pi / 5$ two anticrossings between edge states are marked ( $\square$ and $\bigcirc$ ). The hybridization gap decays exponentially with the number of cells $N_{y}$, and it is barely noticeable for the case shown here. (b) Energy spectrum for $\mathcal{J}_{0}(2 E \delta / \omega)=0.5$. As the bands shrink due to the effective hopping renormalization, two bands of Shockley-like edge states (one for each edge) separate from the upper part of the spectrum. (c) Time evolution of the occupation in the left and right edges of a thin ribbon or cylinder of the Lieb lattice with $N_{y}=5$ unit cells in the $y$ direction. The magnetic flux is $\Phi=\Phi_{0} / 10$, and there is no ac driving. The momentum of the initial state is set to $k_{0}$ with a probability homogeneously distributed among the sites at the left edge (blue in the scheme) and zero elsewhere. In red, the occupancy of the sites at the right edge. The sum of the occupancies on both edges (in gray) remains constant and close to 2 , indicating that the doublon does not occupy sites in the bulk of the lattice.

of an ac driving to independently tune the doublon effective hopping and this local chemical potential. If a lattice contains a sublattice of sites with a certain coordination number, different from the coordination number of the remaining sites, this effect can be harnessed to limit the propagation of the doublon to just that sublattice. In finite samples this effect can also be used to confine the doublon to particular sites at the edges. We also discuss the coexistence of topological and Shockley edge states in 2D systems threaded by a magnetic flux. This coexistence, which does not occur in 1D systems with nontrivial topology, allows the direct doublon transfer between edges in a richer manner than in 1D systems via the coherent superpositions of either Shockley or topological edge states. Our analysis is valid for any 2D lattice and can be investigated experimentally in cold-atom lattices [44] or photonic crystals [45]. Developing 
this work to address a many-particle scenario is an exciting future avenue for research. However, even the two-particle results we report could be of relevance to experimentalists as these effects could be used to distinguish single particles as opposed to doublons in a dilute gas just by looking at its dynamics.

\section{ACKNOWLEDGMENTS}

We would like to thank A. Gómez-León for enlightening discussions. M.B. and G.P. were supported by Spain's MINECO through Grant No. MAT2014-58241-P, and C.E.C. was supported by Grant No. FIS2013-41716-P.

\section{APPENDIX A: EFFECTIVE HAMILTONIAN FOR DOUBLONS}

We start from a Fermi-Hubbard model with an ac field that couples to the particle density and a magnetic flux that induces complex phases in the hoppings. The Hamiltonian of the system is

$$
\begin{aligned}
H(t)= & -J \sum_{\langle i, j\rangle, \sigma} e^{i \phi_{j i}} c_{j \sigma}^{\dagger} c_{i \sigma}+U \sum_{i} n_{i \uparrow} n_{i \downarrow} \\
& +\sum_{i} V_{i}(t)\left(n_{i \uparrow}+n_{i \downarrow}\right) \\
\equiv & H_{J}+H_{U}+H_{A C}(t) .
\end{aligned}
$$

For a time-periodic Hamiltonian, $H(t+T)=H(t)$ with $T=$ $2 \pi / \omega$, Floquet's theorem permits us to write the time-evolution operator $U\left(t_{2}, t_{1}\right)$ as

$$
U\left(t_{2}, t_{1}\right)=e^{-i K\left(t_{2}\right)} e^{-i H_{\mathrm{eff}}\left(t_{2}-t_{1}\right)} e^{i K\left(t_{1}\right)},
$$

where $H_{\text {eff }}$ is a time-independent (effective) Hamiltonian and $K(t)$ is a $T$-periodic self-adjoint operator. $H_{\text {eff governs }}$ the long-term dynamics whereas $e^{-i K(t)}$, also known as the micromotion operator, accounts for the fast dynamics occurring within a period. Following several perturbative methods [46,47], it is possible to find expressions for these operators as power series in $1 / \omega$,

$$
H_{\mathrm{eff}}=\sum_{n=0}^{\infty} \frac{H^{[n]}}{\omega^{n}}, \quad K(t)=\sum_{n=0}^{\infty} \frac{K^{[n]}(t)}{\omega^{n}} .
$$

The different terms in these expansions have a progressively more complicated dependence on the Fourier components of the original Hamiltonian $H^{(q)}=T^{-1} \int_{0}^{T} H(t) e^{i \omega q t} d t$. The first three of them are as follows:

$$
\begin{gathered}
H^{[0]}=H^{(0)}, \quad H^{[1]}=\sum_{q \neq 0} \frac{H^{(-q)} H^{(q)}}{q}, \\
H^{[2]}=\sum_{q, p \neq 0}\left(\frac{H^{(-q)} H^{(q-p)} H^{(p)}}{q p}-\frac{H^{(-q)} H^{(q)} H^{(0)}}{q^{2}}\right) .
\end{gathered}
$$

Before deriving the effective Hamiltonian, it is convenient to transform the original Hamiltonian (A1) into the rotating (a)

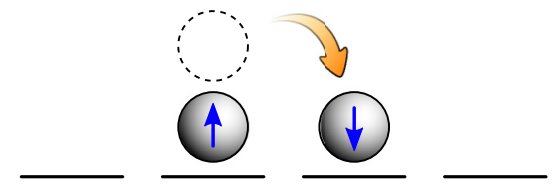

(b)

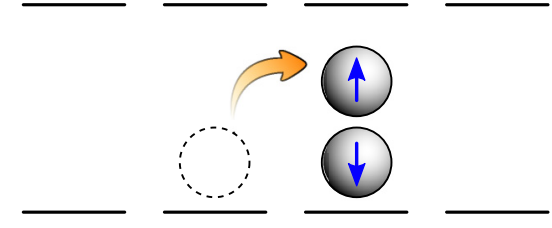

(c)

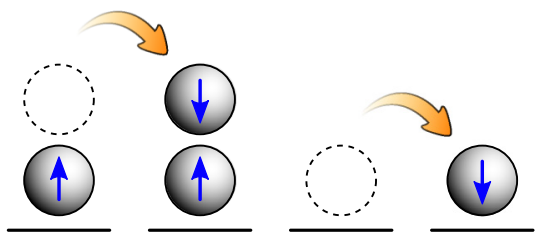

FIG. 8. Schematic of the different hoppings: (a) $h_{i j \sigma}^{-}$, (b) $h_{i j \sigma}^{+}$, and (c) $h_{i j \sigma}^{0}$.

frame with respect to both the interaction and the ac field,

$$
\begin{gathered}
H_{\mathrm{int}}(t)=\mathcal{U}^{\dagger}(t) H(t) \mathcal{U}(t)-i \mathcal{U}^{\dagger}(t) \partial_{t} \mathcal{U}(t), \\
\mathcal{U}(t)=e^{-i H_{U} t-i \int H_{A C}(t) d t} .
\end{gathered}
$$

It can be written as

$$
\begin{aligned}
H_{\mathrm{int}}(t)= & -\sum_{\langle i, j\rangle, \sigma} J e^{i \mathbf{A}(t) \cdot \mathbf{d}_{i j}}\left[1-n_{i \bar{\sigma}}\left(1-e^{i U t}\right)\right] \\
& \times e^{i \phi_{i j}} c_{i \sigma}^{\dagger} c_{j \sigma}\left[1-n_{j \bar{\sigma}}\left(1-e^{-i U t}\right)\right] \\
= & -\sum_{\langle i, j\rangle, \sigma} J e^{i\left[\mathbf{A}(t) \cdot \mathbf{d}_{i j}+\phi_{i j}\right]}\left[h_{i j \sigma}^{0}+e^{i U t} h_{i j \sigma}^{+}+e^{-i U t} h_{i j \sigma}^{-}\right] .
\end{aligned}
$$

Here, we have defined

$$
\begin{gathered}
h_{i j \sigma}^{0}=n_{i \bar{\sigma}} c_{i \sigma}^{\dagger} c_{j \sigma} n_{j \bar{\sigma}}+\left(1-n_{i \bar{\sigma}}\right) c_{i \sigma}^{\dagger} c_{j \sigma}\left(1-n_{j \bar{\sigma}}\right), \\
h_{i j \sigma}^{+}=n_{i \bar{\sigma}} c_{i \sigma}^{\dagger} c_{j \sigma}\left(1-n_{j \bar{\sigma}}\right), \\
h_{i j \sigma}^{-}=\left(h_{j i \sigma}^{+}\right)^{\dagger}=\left(1-n_{i \bar{\sigma}}\right) c_{i \sigma}^{\dagger} c_{j \sigma} n_{j \bar{\sigma}} .
\end{gathered}
$$

The operators $h_{i j \sigma}^{0}$ involve hopping processes that conserve the total double occupancy, whereas $h_{i j \sigma}^{+}$and $h_{i j \sigma}^{-}$raise and lower, respectively, the total double occupancy (see Fig. 8). $\mathbf{A}(t)$ is a vector potential that corresponds to the ac field. In the case of circular polarization, $\mathbf{A}(t)=(\cos \omega t, \sin \omega t) E / \omega ; \mathbf{d}_{i j}=$ $\mathbf{r}_{i}-\mathbf{r}_{j}$ is the vector connecting sites $i$ and $j$. In order to apply the HFE we need to find a common frequency. We will consider first the resonant regime $U=l \omega$ and then, by means of analytical continuation, obtain the strongly interacting limit $(U \gg \omega>J)$ and the high-frequency limit $(\omega \gg U>J)$. The Fourier components of $H_{\text {int }}(t)$ are

$$
H_{\mathrm{int}}^{(q)}=-\sum_{\langle i, j\rangle, \sigma} J_{i j}^{(q)} h_{i j \sigma}^{0}+J_{i j}^{(q+l)} h_{i j \sigma}^{+}+J_{i j}^{(q-l)} h_{i j \sigma}^{-},
$$


where (using the Jacobi-Anger identity),

$$
\begin{aligned}
J_{i j}^{(q)} & =J e^{i \phi_{i j}} e^{-i q \alpha_{i j}} \mathcal{J}_{q}\left(\frac{E \delta}{\omega}\right), \\
J_{j i}^{(q)} & =\left[J_{i j}^{(-q)}\right]^{*} \\
& =J e^{-i \phi_{i j}} e^{-i q \alpha_{i j}} \mathcal{J}_{-q}\left(\frac{E \delta}{\omega}\right),
\end{aligned}
$$

with $\alpha_{i j}=\arctan \left(d_{i j}^{y} / d_{i j}^{x}\right)$ and $\delta=\left|\mathbf{d}_{i j}\right| . \mathcal{J}_{q}$ stands for the Bessel function of the first kind of order $q$.

Now, the zeroth-order approximation in the HFE is given by

$$
\begin{aligned}
H_{\mathrm{int}}^{[0]}= & -J \sum_{\langle i, j\rangle, \sigma} e^{i \phi_{i j}}\left[\mathcal{J}_{0}\left(\frac{E \delta}{\omega}\right) h_{i j \sigma}^{0}\right. \\
& \left.+\mathcal{J}_{l}\left(\frac{E \delta}{\omega}\right) h_{i j \sigma}^{+}+\mathcal{J}_{-l}\left(\frac{E \delta}{\omega}\right) h_{i j \sigma}^{-}\right] .
\end{aligned}
$$

In contrast to the undriven case, the total double occupancy is not an approximate conserved quantity in the strongly interacting limit. There are terms proportional to $\mathcal{J}_{l}\left(\frac{E \delta}{\omega}\right)$ that correspond to the formation and dissociation of doublons assisted by the ac field. However, for low driving amplitudes $(E \delta / \omega<l)$ the probability for these processes to occur is very small, and we can neglect them. It is in this low amplitude regime where it makes sense to consider an effective Hamiltonian for the double-occupancy sector of the space of states. Thus, we will ignore the terms that go with $h_{i j \sigma}^{0}$ because they act nontrivially only on states with some single occupancy.

In the next order of the HFE, there will appear more terms that do not conserve the total double occupancy, which we neglect, and from those which do conserve it, we only keep the ones that act on the doublon's subspace of states,

$$
\begin{aligned}
\frac{H_{\mathrm{int}}^{[1]}}{\omega} \simeq & \sum_{\langle i, j\rangle, \sigma}\left[\frac{1}{\omega} \sum_{q \neq 0} \frac{\mathcal{J}_{-q+l}^{2}\left(\frac{E \delta}{\omega}\right) J^{2} h_{i j \sigma}^{+} h_{j i \sigma}^{-}}{q}\right. \\
& \left.+\frac{1}{\omega} \sum_{q \neq 0} \frac{\mathcal{J}_{-q+l}\left(\frac{E \delta}{\omega}\right) \mathcal{J}_{q-l}\left(\frac{E \delta}{\omega}\right) J^{2} e^{i 2 \phi_{i j}} h_{i j \sigma}^{+} h_{i j \bar{\sigma}}^{-}}{q}\right] .
\end{aligned}
$$

Here, the first term is equal to

$$
\begin{aligned}
\frac{J^{2}}{\omega} \sum_{p \neq-l} \frac{\mathcal{J}_{p}^{2}\left(\frac{E \delta}{\omega}\right) h_{i j \sigma}^{+} h_{j i \sigma}^{-}}{p+l}= & \frac{J^{2}}{U} \sum_{p \neq-l} \frac{\mathcal{J}_{p}^{2}\left(\frac{E \delta}{\omega}\right)}{p \omega / U+1} \\
& \times\left(n_{i \bar{\sigma}} n_{i \sigma}-n_{i \bar{\sigma}} n_{i \sigma} n_{j \sigma} n_{j \bar{\sigma}}\right),
\end{aligned}
$$

and the second term is equal to

$$
\begin{aligned}
& \frac{J^{2} e^{i 2 \phi_{i j}}}{\omega} \sum_{p \neq-l} \frac{\mathcal{J}_{p}\left(\frac{E \delta}{\omega}\right) \mathcal{J}_{-p}\left(\frac{E \delta}{\omega}\right) h_{i j \sigma}^{+} h_{j i \sigma}^{-}}{p+l} \\
& =\frac{J^{2} e^{i 2 \phi_{i j}}}{U} \sum_{p \neq-l} \frac{\mathcal{J}_{p}\left(\frac{E \delta}{\omega}\right) \mathcal{J}_{-p}\left(\frac{E \delta}{\omega}\right)}{p \omega / U+1} c_{i \sigma}^{\dagger} c_{i \bar{\sigma}}^{\dagger} c_{j \bar{\sigma}} c_{j \sigma} .
\end{aligned}
$$

In the limit $U \gg \omega>J, p \omega / U \ll 1$, we can approximate all the denominators in the above expressions as 1 . Also, when analytically continuing the formulas for values of $U$ other than multiples of the frequency, the restriction $p \neq-l$ has no meaning. Finally, using the identities,

$$
\begin{aligned}
\sum_{q=-\infty}^{\infty} \mathcal{J}_{q}^{2}(\alpha) & =1, \\
\sum_{q=-\infty}^{\infty} \mathcal{J}_{q}(\alpha) \mathcal{J}_{k-q}(\beta) & =\mathcal{J}_{k}(\alpha+\beta),
\end{aligned}
$$

we arrive at

$$
\begin{aligned}
H_{\mathrm{eff}}^{U \gg \omega} & =J_{\mathrm{eff}} \sum_{\langle i, j\rangle} e^{i 2 \phi_{i j}} d_{i}^{\dagger} d_{j}+\sum_{i} \mu_{i} n_{i}^{d}-\frac{2 J^{2}}{U} \sum_{\langle i, j\rangle} n_{i}^{d} n_{j}^{d}, \\
J_{\mathrm{eff}} & \equiv 2 J^{2} \mathcal{J}_{0}\left(2 \frac{E \delta}{\omega}\right) / U, \quad \mu_{i} \equiv 2 J^{2} z_{i} / U .
\end{aligned}
$$

Here we have expressed the effective Hamiltonian in terms of the doublon creation and annihilation operators $d_{i}^{\dagger}=c_{i \uparrow}^{\dagger} c_{i \downarrow}^{\dagger}$ and $d_{i}=c_{i \downarrow} c_{i \uparrow}$ and the doublon number operator $n_{i}^{d}=$ $d_{i}^{\dagger} d_{i} ; z_{i}$ is the number of neighbors of site $i$. Importantly, there is a term that corresponds to the attractive interaction between neighboring doublons, but since we only have one doublon in the system, we do not take it into account.

For completeness we give also the result in the other limit: $\omega \gg U>J$. Now $p \omega / U$ is very large, and all the terms in the sums are very small except those for $p=0$. The effective Hamiltonian in this case would be as follows:

$$
\begin{gathered}
H_{\mathrm{eff}}^{\omega \gg U}=J_{\mathrm{eff}} \sum_{\langle i, j\rangle} e^{i 2 \phi_{i j}} d_{i}^{\dagger} d_{j}+\sum_{i} \mu_{i} n_{i}^{d}-J_{\mathrm{eff}} \sum_{\langle i, j\rangle} n_{i}^{d} n_{j}^{d}, \\
J_{\mathrm{eff}} \equiv 2 J^{2} \mathcal{J}_{0}^{2}\left(\frac{E \delta}{\omega}\right) / U, \quad \mu_{i} \equiv J_{\mathrm{eff}} z_{i} .
\end{gathered}
$$

It is worth mentioning that these results could also be obtained by applying the HFE sequentially, integrating first the fast varying terms corresponding to the leading energy scale in the system [33]. We also note that higher-order corrections will include complex next-nearest-neighbor hoppings that break the time-reversal symmetry in systems without the presence of a magnetic flux. Nonetheless, we expect them not to be very significant for the effects of sublattice and edge confinements discussed in the main text.

\section{APPENDIX B: TIME AVERAGE AND STANDARD DEVIATION}

According to the definition, the probability $p_{A}(t)$ is $p_{A}(t)=$ $\left\|U_{A}(t)\right\|^{2} / N_{A}$, where $\|\cdot\|$ denotes the Hilbert-Schmidt norm and $U_{A}(t)=P_{A} U(t) P_{A}$ is the time-evolution operator projected on the subspace of the $A$ sublattice. Using the spectral 
decomposition,

$$
U(t)=\sum_{\mathbf{k}} \sum_{n} e^{-i \epsilon_{n}(\mathbf{k}) t}\left|u_{\mathbf{k}}^{n}\right\rangle\left\langle u_{\mathbf{k}}^{n}\right|, \quad n \in\{0, \pm\},
$$

we can express

$$
\begin{aligned}
\left\|U_{A}(t)\right\|^{2}= & \sum_{\mathbf{k}}\left|\frac{e^{-i \epsilon_{+}(\mathbf{k}) t}}{1+g_{+}(\mathbf{k})}+\frac{e^{-i \epsilon_{-}(\mathbf{k}) t}}{1+g_{-}(\mathbf{k})}\right|^{2} \\
= & \sum_{\mathbf{k}}\left[\frac{1}{1+g_{+}(\mathbf{k})}\right]^{2}+\left[\frac{1}{1+g_{-}(\mathbf{k})}\right]^{2} \\
& +\frac{2 \cos \left(\epsilon_{+} t-\epsilon_{-} t\right)}{\left[1+g_{+}(\mathbf{k})\right]\left[1+g_{-}(\mathbf{k})\right]}
\end{aligned}
$$

where we have defined $g_{+}(\mathbf{k})=\frac{\left|f_{1}(\mathbf{k})\right|^{2}+\left|f_{2}(\mathbf{k})\right|^{2}}{\epsilon_{+}^{2}(\mathbf{k})}$ and $g_{-}(\mathbf{k})=\frac{\left|f_{1}(\mathbf{k})\right|^{2}+\left|f_{2}(\mathbf{k})\right|^{2}}{\epsilon_{-}^{2}(\mathbf{k})}$. The time average is given by

$$
\overline{p_{A}}=\lim _{t \rightarrow \infty} \frac{1}{t} \int_{0}^{t} p_{A}\left(t^{\prime}\right) d t^{\prime}
$$

$$
\simeq \frac{1}{V} \int_{\mathrm{FBZ}}\left[\frac{1}{1+g_{+}(\mathbf{k})}\right]^{2}+\left[\frac{1}{1+g_{-}(\mathbf{k})}\right]^{2} d \mathbf{k} .
$$

Here $V$ stands for the area of the first Brillouin zone (FBZ). The value of this integral as a function of $r=\Delta \mu / J_{\text {eff }}$ is shown in Fig. 2 in the main article. In a similar way we can compute the variance of $p_{A}$ as

$$
\sigma_{A}^{2}=\overline{p_{A}^{2}}-{\overline{p_{A}}}^{2},
$$

with

$$
\begin{gathered}
\overline{p_{A}^{2}}=\lim _{t \rightarrow \infty} \frac{1}{t} \int_{0}^{t}\left[p_{A}\left(t^{\prime}\right)\right]^{2} d t^{\prime}, \\
\sigma_{A}^{2} \simeq \frac{1}{V^{2}} \int_{\mathrm{FBZ}} \frac{2}{\left[1+g_{+}(\mathbf{k})\right]^{2}\left[1+g_{-}(\mathbf{k})\right]^{2}} d \mathbf{k} .
\end{gathered}
$$

When the magnetic flux is not zero, the calculation is more involved since it is necessary to take into account the larger magnetic unit cell.
[1] D. Guzmán-Silva, C. Mejía-Cortés, M. A. Bandres, M. C. Rechtsman, S. Weimann, S. Nolte, M. Segev, A. Szameit, and R. A. Vicencio, New J. Phys. 16, 063061 (2014).

[2] S. Mukherjee, A. Spracklen, D. Choudhury, N. Goldman, P. Öhberg, E. Andersson, and R. R. Thomson, Phys. Rev. Lett. 114, 245504 (2015).

[3] S. Mukherjee and R. R. Thomson, Opt. Lett. 40, 5443 (2015).

[4] L. Sansoni, F. Sciarrino, G. Vallone, P. Mataloni, A. Crespi, R. Ramponi, and R. Osellame, Phys. Rev. Lett. 108, 010502 (2012).

[5] J. R. Petta, A. C. Johnson, J. M. Taylor, E. A. Laird, A. Yacoby, M. D. Lukin, C. M. Marcus, M. P. Hanson, and A. C. Gossard, Science 309, 2180 (2005).

[6] L. Gaudreau, G. Granger, A. Kam, G. C. Aers, S. A. Studenikin, P. Zawadzki, M. Pioro-Ladriere, Z. R. Wasilewski, and A. S. Sachrajda, Nat. Phys. 8, 54 (2012).

[7] F. Forster, G. Petersen, S. Manus, P. Hänggi, D. Schuh, W. Wegscheider, S. Kohler, and S. Ludwig, Phys. Rev. Lett. 112, 116803 (2014).

[8] I. Bloch, J. Dalibard, and W. Zwerger, Rev. Mod. Phys. 80, 885 (2008).

[9] M. Atala, M. Aidelsburger, J. T. Barreiro, D. Abanin, T. Kitagawa, E. Demler, and I. Bloch, Nat. Phys. 9, 795 (2013).

[10] T. Li, L. Duca, M. Reitter, F. Grusdt, E. Demler, M. Endres, M. Schleier-Smith, I. Bloch, and U. Schneider, Science 352, 1094 (2016).

[11] L.-K. Lim, T. Troppenz, and C. Morais Smith, Phys. Rev. A 84, 053609 (2011).

[12] R. Sánchez, G. Granger, L. Gaudreau, A. Kam, M. PioroLadrière, S. A. Studenikin, P. Zawadzki, A. S. Sachrajda, and G. Platero, Phys. Rev. Lett. 112, 176803 (2014).

[13] S. Bose, Phys. Rev. Lett. 91, 207901 (2003).

[14] M.-H. Yung and S. Bose, Phys. Rev. A 71, 032310 (2005).

[15] A. Benseny, J. Gillet, and T. Busch, Phys. Rev. A 93, 033629 (2016).

[16] D. H. Dunlap and V. M. Kenkre, Phys. Rev. B 34, 3625 (1986).

[17] M. Grifoni and P. Hänggi, Phys. Rep. 304, 229 (1998).
[18] N. Goldman, G. Juzeliūnas, P. Öhberg, and I. B. Spielman, Rep. Prog. Phys. 77, 126401 (2014).

[19] C. E. Creffield and F. Sols, Phys. Rev. A 90, 023636 (2014).

[20] J. Struck, C. Ölschläger, R. Le Targat, P. Soltan-Panahi, A Eckardt, M. Lewenstein, P. Windpassinger, and K. Sengstock, Science 333, 996 (2011).

[21] G. Jotzu, M. Messer, R. Desbuquois, M. Lebrat, T. Uehlinger, D. Greif, and T. Esslinger, Nature (London) 515, 237 (2014).

[22] K. Winkler, G. Thalhammer, F. Lang, R. Grimm, J. Hecker Denschlag, A. J. Daley, A. Kantian, H. P. Büchler, and P. Zoller, Nature (London) 441, 853 (2006).

[23] N. Strohmaier, D. Greif, R. Jördens, L. Tarruell, H. Moritz, T. Esslinger, R. Sensarma, D. Pekker, E. Altman, and E. Demler, Phys. Rev. Lett. 104, 080401 (2010).

[24] P. M. Preiss, R. Ma, M. E. Tai, A. Lukin, M. Rispoli, P. Zupancic, Y. Lahini, R. Islam, and M. Greiner, Science 347, 1229 (2015).

[25] E. Compagno, L. Banchi, C. Gross, and S. Bose, Phys. Rev. A 95, 012307 (2017).

[26] S. Folling, S. Trotzky, P. Cheinet, M. Feld, R. Saers, A. Widera, T. Muller, and I. Bloch, Nature (London) 448, 1029 (2007).

[27] U. Schneider, L. Hackermuller, J. P. Ronzheimer, S. Will, S. Braun, T. Best, I. Bloch, E. Demler, S. Mandt, D. Rasch, and A. Rosch, Nat. Phys. 8, 213 (2012).

[28] C. E. Creffield and G. Platero, Phys. Rev. Lett. 105, 086804 (2010).

[29] C. E. Creffield and G. Platero, Phys. Rev. B 69, 165312 (2004).

[30] F. Hofmann and M. Potthoff, Phys. Rev. B 85, 205127 (2012).

[31] M. Bukov, L. D’Alessio, and A. Polkovnikov, Adv. Phys. 64, 139 (2015).

[32] M. Bello, C. E. Creffield, and G. Platero, Sci. Rep. 6, 22562 (2016).

[33] M. Bukov, M. Kolodrubetz, and A. Polkovnikov, Phys. Rev. Lett. 116, 125301 (2016).

[34] W. Beugeling, J. C. Everts, and C. Morais Smith, Phys. Rev. B 86, 195129 (2012). 
[35] J. Vidal, R. Mosseri, and B. Douçot, Phys. Rev. Lett. 81, 5888 (1998).

[36] O. Mülken and A. Blumen, Phys. Rep. 502, 37 (2011).

[37] J. Vidal, P. Butaud, B. Douçot, and R. Mosseri, Phys. Rev. B 64, 155306 (2001).

[38] B. A. Bernevig and T. L. Hughes, Topological Insulators and Topological Superconductors (Princeton University Press, Princeton, 2013).

[39] S. Ryu, A. P. Schnyder, A. Furusaki, and A. W. W. Ludwig, New J. Phys. 12, 065010 (2010).

[40] E. V. Castro, M. P. López-Sancho, and M. A. H. Vozmediano, Phys. Rev. B 92, 085410 (2015).

[41] E. Prodan, T. L. Hughes, and B. A. Bernevig, Phys. Rev. Lett. 105, 115501 (2010).
[42] M. Aidelsburger, M. Atala, M. Lohse, J. T. Barreiro, B. Paredes, and I. Bloch, Phys. Rev. Lett. 111, 185301 (2013).

[43] H. Miyake, G. A. Siviloglou, C. J. Kennedy, W. C. Burton, and W. Ketterle, Phys. Rev. Lett. 111, 185302 (2013).

[44] S. Taie, H. Ozawa, T. Ichinose, T. Nishio, S. Nakajima, and Y. Takahashi, Sci. Adv. 1, e1500854 (2015).

[45] S. Mukherjee, M. Valiente, N. Goldman, A. Spracklen, E. Andersson, P. Öhberg, and R. R. Thomson, Phys. Rev. A 94, 053853 (2016).

[46] A. Eckardt and E. Anisimovas, New J. Phys. 17, 093039 (2015).

[47] T. Mikami, S. Kitamura, K. Yasuda, N. Tsuji, T. Oka, and H. Aoki, Phys. Rev. B 93, 144307 (2016). 